\title{
Archives et vestiges de la grotte du château de Noisy
}

Archives and remains of the grotto of the chateau de Noisy

\section{Bruno Bentz}

\section{(2) OpenEdition}

1 Journals

Édition électronique

URL : http://journals.openedition.org/artefact/5461

DOI : 10.4000/artefact.5461

ISSN : 2606-9245

Éditeur :

Association Artefact. Techniques histoire et sciences humaines, Presses universitaires du Midi

\section{Édition imprimée}

Date de publication : 15 juillet 2020

Pagination : 89-108

ISBN : 978-2-8107-0691-4

ISSN : 2273-0753

\section{Référence électronique}

Bruno Bentz, "Archives et vestiges de la grotte du château de Noisy », Artefact [En ligne], 12 | 2020, mis en ligne le 21 décembre 2020, consulté le 23 décembre 2020. URL : http://

journals.openedition.org/artefact/5461; DOI : https://doi.org/10.4000/artefact.5461

\section{(c) (i) (9)}

Artefact, Techniques, histoire et sciences humaines est mise à disposition selon les termes de la Licence Creative Commons Attribution - Pas d'Utilisation Commerciale - Pas de Modification 4.0 International. 


\section{Archives et vestiges de la grotte du château de Noisy}

\section{Bruno Bentz}

\section{Résumé}

La grotte du château de Noisy a été bâtie en 1582 pour Albert de Gondi. Elle se trouvait au rez-de-chaussée d'un grand pavillon au cœur d'un vaste jardin en terrasses, avant d'être démolie en 1732. Plusieurs témoignages en ont gardé le souvenir, notamment des gravures de Jean Marot, une longue description et une série de plans datant de la fin du xVII e siècle, à l'époque où la propriété avait été acquise par Louis XIV. Intégré au domaine royal dans la forêt de Marly, le site a été préservé. Des fouilles archéologiques ont pu y être entreprises en 2017 et 2019. Elles ont mis au jour les fondations de la grotte et une partie des décors, encore en place ou mêlés à la couche de remblais issus de la démolition. Ces décors associent, dans des supports en plâtre, une grande variété de rocailles et de coquilles, ainsi que des éléments dorés et sculptés. L'analyse des archives et des vestiges met en évidence la richesse et la complémentarité des sources pour retracer l'histoire et les conditions de la fabrication de ce qui fut probablement un chef-d'œuvre d'architecture et de décoration de la Renaissance.

\section{Mots-clés}

rocailles, coquilles, sculpture, hydraulique, fouilles archéologiques, Jean Marot, Gondi

99 Bruno Bentz, «Archives et vestiges de la grotte du château de Noisy », Artefact, 12, 2020, p. 89-108. 


\section{Archives and remains of the grotto of the chateau de Noisy}

\section{Abstract}

The grotto of the chateau de Noisy was built in 1582 for Albert de Gondi. It was located on the ground floor of a large pavilion in the centre of a wide terraced garden, before being demolished in 1732. Several evidences have been preserved, including engravings by Jean Marot, a long description and a series of plans dating from the end of the $17^{\text {th }}$ century, when the property was acquired by Louis XIV. Integrated into the royal domain in the Marly forest, the site has been preserved. Archaeological digs could be undertaken in 2017 and 2019. They revealed the foundations of the grotto and part of the decorations, still in place or mixed with the layer of embankments resulting from the demolition. These decorations combine, in plaster supports, a wide variety of rockeries and shells, as well as gilded and sculpted elements. The analysis of the archives and remains highlights the abundance and complementarity of the sources to trace the history and conditions of the making of what was probably a masterpiece of Renaissance architecture and decoration.

\section{Keywords}

rockery, shell, sculpture, hydraulics, archaeological digs, Jean Marot, Gondi 
$\grave{A}$ la suite des fouilles archéologiques réalisées en 2017 et 2019'1 , l'architecture et la décoration de la grotte aménagée pour Albert de Gondi à Noisy sont désormais beaucoup mieux connues. Bâtie en 1582 dans les jardins du château, la grotte fut démolie en 1732. Albert de Gondi, d'origine florentine, était maréchal de France et, à la suite de son mariage avec Catherine de Clermont, il devint duc de Retz. Proche de la reine-mère et du roi Henri III, il acquit les seigneuries de Noisy et des environs de Saint-Germain-en-Laye où résidait régulièrement la cour de France. Conçu comme une villa italienne, le château de Noisy était entouré de jardins en terrasses agrémentés de fontaines. La grotte avait été aménagée au rez-de-chaussée d'un pavillon relié à la terrasse du château par une galerie d'arcades d'une centaine de mètres de longueur. C'était probablement l'élément le plus original de la demeure, dont la magnificence était destinée à émerveiller le roi lorsqu'il se rendait à Noisy, comme le feront ses successeurs jusqu'au règne de Louis XIV qui acquit le domaine un siècle plus tard.

Abandonné au début du XviII ${ }^{\mathrm{e}}$ siècle puis détruit, le château de Noisy a été en grande partie intégré à la forêt de Marly (aujourd'hui forêt domaniale) et, pour cette raison, il a été préservé de l'urbanisation. Longtemps oublié sous la végétation, le site est repéré à la fin $\mathrm{du} \mathrm{XIX}^{\mathrm{e}}$ siècle par le dessinateur Auguste Guillaumot et par les historiens, Adrien Maquet et Albert Terrade. Dès cette époque, les principales sources d'archives sont identifiées : la description de Boulin, les gravures de Marot, les plans des jardins ${ }^{2}$. Les premières recherches sur le terrain eurent lieu à la même époque et un érudit local, F. Prodhomme, parvint « à déterminer très exactement l'emplacement qu'occupait la grotte ${ }^{3} »$.

Ainsi, de nombreuses sources sont disponibles pour étudier la grotte de Noisy. C'est dans ces conditions que l'archéologie de l'époque moderne se justifie pleinement. La documentation et les ruines apportent des indices

1. Grotte du château de Noisy (Bailly, Yvelines), fouilles réalisées avec le concours de la Direction régionale des affaires culturelles d'Île-de-France (Service régional de l'archéologie) et de l'Office national des forêts (Agence territoriale Île-de-France Ouest), avec le soutien des villes de Noisyle-Roi et de Bailly, et le concours de l'Association Renaissance du Patrimoine de Noisy-le-Roi Rennemoulin Bailly, de l'association Volutes, de l'association OMAGE et du Musée national de le Renaissance (Écouen).

2. Les sources sont citées dans le texte et référencées en annexe.

3. Terrade, 1899, p. 83. 
et des traces qu'il convient d'analyser et de critiquer en s'appuyant sur la totalité des données, les archives et les vestiges s'enrichissant mutuellement, l'abondance n'étant jamais de trop. En effet, les plans, les vues et les descriptions anciennes ne rendent que très partiellement compte de la réalité tandis que les découvertes du bâti et de son ornementation n'expliquent pas tout de l'ancienne construction. C'est la raison pour laquelle il convient de mener conjointement l'enquête et l'investigation avant d'établir un premier bilan archéologique.

\section{Les archives}

L'enquête s'appuie d'abord sur les sources citées dans la bibliographie, l'étude la plus complète étant celle publiée en 1977 par Hélène Couzy, fondée sur les archives mais envisageant aussi la possibilité de faire des fouilles archéologiques ${ }^{4}$. Les sources relatives à la période de construction et d'utilisation de la grotte au temps d'Albert de Gondi, mort en 1602, sont peu nombreuses. En juillet 1599, un voyageur morave, Zdenek Waldstein, vient à Noisy et atteste dans son journal l'existence de la grotte: "[ hortus] in eo vidimus elegantem fontem ex conchis, cochleis paratum " [un jardin dans lequel nous avons vu une belle fontaine ornée de coquilles d'escargots], mais c'est une lettre de $1582^{6}$ qui apporte les plus anciennes indications sur la réalisation de la grotte. Ce document manuscrit a été rédigé en italien par Andréa Albertani pour le grand-duc de Toscane, François Ir de Médicis, dont il était l'ambassadeur extraordinaire en France, mais il a été traduit et publié avec des lacunes en $1953^{7}$ et repris ensuite sans être vérifié. Le texte original, publié récemment ${ }^{8}$, a permis de relever deux

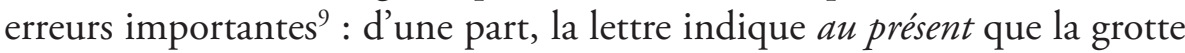
" a d'innombrables niches ", ce qui prouve que la construction était commencée ; d'autre part, la lettre rapporte que Gondi sollicite le concours d'un artisan travaillant " à Pratolino " pour achever la décoration de la

\footnotetext{
4. Couzy, 1977 : "Tous ces vestiges sont à fleur de terre et il semble qu'une fouille menée à cet emplacement permettrait de mettre à jour les fondations " (p. 32).

5. Vatican, Reg.lat.666, fol. 58r ; publié par Odier, 1926, p. 169 ; cité par Cousy, 1977, p. 30 et note 34 .

6. Florence, Archivio di Stato, $\mathrm{n}^{\circ} 4611$, fol. 113 bis.

7. Jullien de Pommerol, 1953, p. 149.

8. Calafati, 2015, t. I p. 429 ; Cicali, 2017, p. 365 n. 22.

9. Bentz, 2018b, p. 126.
} 
grotte. Il n'est pas étonnant que la grotte soit en construction dès $1582 \mathrm{car}$ les travaux de construction du château et des jardins avaient commencé vers 1575 . Le pavillon de la grotte est imbriqué dans le système des terrasses et relié par des arcades : il a nécessairement été conçu dès l'origine. Le rapprochement avec les grottes de Pratolino est également essentiel car il permet de comprendre le système décoratif adopté. La datation des travaux autour de l'année 1582 a notamment pour effet d'exclure une intervention de Tomaso Francini arrivé en France, à la demande de Henri IV, en 1599 pour la réalisation des grottes du Château-Neuf de Saint-Germain-enLaye. Cette lettre signale aussi que Gondi a été le commanditaire de la grotte, sans mentionner explicitement qu'il s'agit de Noisy mais simplement qu'elle se trouve "dans sa belle propriété ». Cependant, il ne peut s'agir de son hôtel parisien, de plus petites dimensions et pour lequel il avait déjà réalisé une grotte quelques années plus tôt en $1578^{10}$.

Les sources textuelles sont plus nombreuses à partir du début du XvII ${ }^{\mathrm{e}}$ siècle. Le système hydraulique de Noisy est mentionné en 1605 par l'historien Pierre Matthieu, sans citer la grotte ${ }^{11}$. Il rapporte l'invention d'une machine par Claude de Monconis pour élever les eaux dans une fontaine : il est probable que cela concerne le mécanisme du jet d'eau de la grotte décrit plus tard et dont des vestiges ont été retrouvés. En outre, un pavillon de la machine est mentionné à la fin du XVII ${ }^{\mathrm{e}}$ siècle et une pompe est réparée par le fontainier du roi Nicolas Le Jongleur : il doit s'agir de l'installation d'origine pour créer un jet de près de 8 mètres de hauteur qui s'élançait depuis une vasque à travers la voûte de l'abside nord de la grotte pour sortir au milieu des parterres ${ }^{12}$. Ce dispositif n'est pas mentionné dans le Journal de Jean Héroard, en $1607^{13}$, lorsqu'il rapporte à plusieurs reprises la visite du dauphin Louis, âgé de cinq et bientôt six ans, qui manifeste cependant un grand intérêt à se rendre dans la grotte. Au-delà de l'anecdote, ce document apporte trois informations essentielles : d'abord, on apprend que l'enfant aime monter à l'étage ${ }^{14}$, ce qui prouve bien évidemment son existence, après quoi on ne peut accorder aucun crédit à un dessin conservé

10. Bentz, 2020 (à paraître).

11. Mathieu, 1605, p. 265 ; Bentz 2019, p. 116 et n. 6.

12. Bentz, 2018a, p. 91.

13. Le manuscrit du journal est conservé à la Bibliothèque nationale de France (Paris); l'édition de Soulié et Barthélemy de 1868 est incomplète, voir Foisil, 1989, vol. 1, p. 1282-1339.

14. "Mené aux parterres du costé de la grotte ; se joue dans la sale qui est dessus, sort, entre, courant, dict qu'il va a Paris, n’en peult plus partir »; Foisil, 1989, 10 septembre 1607. 
à Stockholm proposé comme un état initial de la grotte. En effet, aucune indication ne permet de supposer que Albert de Gondi ait modifié une grotte déjà réalisée, d'autant que l'étage est parfaitement adapté au niveau supérieur du jardin haut de la grotte sur lequel il est de plain-pied. Ensuite, Héroard fait état de coquilles que l'enfant s'amuse à récupérer de la grotte et à remonter dans sa chambre, ce qui confirme la présence - et la fragilité ! - du décor de la grotte ${ }^{15}$. Enfin, Héroard mentionne l'existence d'une " grotte sèche » située à proximité du château ${ }^{16}$. Il s'agit d'une autre grotte, aménagée sous la rampe menant à la forêt, peu documentée par ailleurs sinon par une vue en coupe de la fin du XVII ${ }^{\mathrm{e}}$ siècle. Son existence souligne une nouvelle fois l'importance accordée aux grottes par Gondi.

Il peut sembler étrange qu'à l'époque de Louis XIV, la grotte soit peu documentée : elle est brièvement mentionnée en 1676 dans l'acte d'acquisition à propos du jardin où « il y a des fontaines jaillissantes en façon de grotte $^{17}$ " puis plus aucune mention n'est signalée, ni pour un entretien ou une réparation, ni par un visiteur, malgré la présence durant quelques années de l'école pour jeunes filles de $\mathrm{M}^{\mathrm{me}}$ de Maintenon ou des petits-enfants du roi. Seule exception, le dessin de Christoph Pitzler, un architecte allemand de passage en France, mais il a manifestement copié la gravure de Marot pour le plan et sa vue de la « fontaine en glacis ou la grote de rocaille " ne correspond pas aux autres sources ${ }^{18}$. Dans le livre de Ernest Tambour ${ }^{19}$ sur le château de Noisy, une citation non référencée pourrait provenir d'un témoignage de cette époque, mais elle ne révèle rien d'original. Certes, le traité de jardinage par Leblond et Dezallier indique en 1709 que " les grotes ne sont maintenant presque plus à la mode ${ }^{20}$ ", ce qui peut expliquer ce long silence des textes, brisé toutefois au dernier moment, lors de la démolition en 1732, par la longue description de François-Bernard Boulin, seigneur de Bailly. Il existe deux copies de ce

15. "Monte en la grotte où il s'amuse a recueillir des cailloux, des coquilles tombées pour en faire une petite dans sa chambre »; Foisil, 1989, 29 août 1607.

16. "Mené dehors, s'amuse à la petite grotte sèche a l'entrée du parc "; Foisil, 1989, 8 septembre 1607.

17. Archives nationales (Paris), $\mathrm{O}^{1} 3932^{\mathrm{A}}$, p. 3.

18. Potsdam, Christoph Pitzler, Reisetagebuch [Carnet de voyage], 1686, fol. 186 ; Couzy, 1977, p. 30 (repr.).

19. Tambour, 1925 , p. 375 : « une belle grotte revêtue de coquillages ou plusieurs figures de la fable étoient représentées ".

20. Leblond-Dezallier, 1709, p. 73. 
manuscrit, ne variant que sur quelques détails, toutefois le texte original est celui conservé par la Bibliothèque municipale de Versailles, annoté par l'auteur en 1735 peu après son achèvement ${ }^{21}$. Ce texte comporte de nombreux détails, sur les dimensions, sur l'architecture et la distribution intérieure, sur la décoration et les matériaux. Comme le rappelaient Leblond et Dezallier, les grottes "sont fort sujettes à se gâter ${ }^{22}$ ", mais Boulin ne signale aucune dégradation, aucun dommage, ni aucune réparation que ses cent cinquante années d'existence avaient nécessairement suscités. La description est très détaillée car elle contient de nombreuses indications techniques, tant sur les mesures, les matériaux que la configuration du pavillon et de ses décors :

Mais rien n'étoit comparable à la grotte qui étoit placée sur la première terrasse d'en bas à la gauche du château du côté de Vaucheron. Elle répondoit au milieu du Boulingrin qui étoit au dessous de cette terrasse, sur laquelle on montoit par un grand perron rond, de dix-huit marches de pierre de tailles qui étoit au devant de cette grotte, dont la façade avait trente pieds de long, quatre belles colonnes de pierre d'ordre composite de quinze pieds de haut chacune y compris les pieds d'estaux, soutenoient une frise avec un fronton. Il y avait dans la frise des faisceaux / d'armes dans lesquels on voyoit des boucliers chargés des armes de Gondy, et dans le fronton deux figures en bas relief à demy couchées représentant l'une une Neptune, et l'autre la déesse Thetis. Toute cette façade étoit terminée par le haut d'un rang de balustres de pierres avec une tablette qui servoit d'appuy à une terrasse qui étoit au-dessus.

La porte d'entrée de cette grotte étoit ornée de refends et fermée d'une grille de fer. Sa profondeur depuis l'entrée jusqu'au fond étoit de huit toises, et la croisée qui la traversoit en avait neuf et demy, à chaque bout de laquelle on trouvoit un petit cabinet éclairé par un portique / grillé qui donnoit sur la terrasse. Au milieu de cette croisée étoit un sallon octogone de vingt pieds de diamètre qui étoit voûté en dôme et qui avoit dix-sept a dix-huit

21. Versailles, Bibliothèque municipale, G 280 (description de la grotte, p. 111-116). Une copie de ce manuscrit est conservée à la Bibliothèque nationale de France (Paris), département des manuscrits, Fr. 11651, p. 72-92 (description de la grotte, p. 85-89) ; publié par Maquet, 1878, p. 366368 ; Terrade, 1899, p. 8-9. Le texte original est reproduit ci-dessous.

22. Leblond-Dezallier, 1709, p. 73. 
pieds de haut. On entroit dans ce sallon par quatre portiques de unze pieds de haut. Tout le dedans de ce sousterrain étoit incrusté de rocailles et de coquillages variés en cent façons différentes formant des compartiments et des mosä̈ques charmantes. Il y avoit dans les quatre pans coupés de ce salon des niches de rocaille et de coquilles dans lesquelles on voyait des figures de tritons et de sirennes faites de petits coquillages qui imitoient parfaitement le naturel. Le plafond du dôme étoit divisé en huit parties égales par arcs surbaissés qui naissaient de chaque angle du salon et qui venoient se réunir au / centre d'où pendoit un gros cul de lampe travaillé à jour d'un goût très recherché. Tous ces ornements étoit faits de coquilles et de rocailles. Le pavé de ce sallon et de toute la grotte étoit de pierre de liais cizelés par compartiments qui répondoient au dessein du plafond, et l'on avoit incrusté dans la pierre des millions de petites pierres noires rondes et grosses au plus comme des noix muscades qui faisoient le fonds de cette mosaïque, et qui étoient tellement unies et attachées ensemble par un mastic si solide qu'on n'en pouvait arracher une. Les deux croisées des côtés et les deux petits cabinets des bouts étoient incrustés de pareils ornements que le sallon. On avoit appliqué contre les murailles, dans des cadres de rocaille de coquillages, divers monstres marins avec des oiseaux aquatiques en bas reliefs faits de coquilles imitant la couleur de leurs écailles et de leur plumage. Il y avait dans le fond / de cette grotte une espèce de buffet de rocaille et de coquilles avec deux dauphins sur les côtés qui jettoient de l'eau dans une grande coquille de pierre qui retomboit ensuite en nape dans un bassin au-dessous. On avait pratiqué en cet endroit une ouverture ronde à la voûte qui donnoit au milieu d'un grand sallon au dessus. Cette ouverture étoit entourée d'une balustrade de fer par le dedans de ce sallon, qui étoit fait à l'italienne et dont les murailles, ainsi que le plafonds étoient peints à fresque, et sur lesquels on avoit représenté des chasses d'animaux. Il étoit beaucoup plus long que large et percé de croisées de tous côtés dont trois en forme de portique étoient placés dans le bout et servoient d'entrée à la terrasse qui faisait le dessus du frontispice de la grotte. En un mot, tout faisoit assés connoitre en cet endroit la magnificence du maitre qui l'avoit fait construire et qui avoit fait venir d'Italie les plus habiles maitres dans ce genre, 
n'ayant rien espargné pour la dépense de cette / grotte qui étoit unique en son espèce et qu'on dit avoir couté cinquante mille écus et avoir été plusieurs années à mettre dans sa perfection ${ }^{23}$.

Cette description apporte donc un état général, mais inévitablement incomplet. Quelques indications de Boulin sur les créateurs italiens ou le coût de la construction de la grotte reposent nécessairement sur une tradition orale (ou des documents inconnus). Certains passages peuvent être confrontés, pour valider leur exactitude, aux archives graphiques et aux vestiges mis au jour. Curieusement, la description est fautive quand Boulin décrit la sortie du jet d'eau dans le salon de l'étage. C'était en réalité dans le parterre voisin, mais sa formulation semble avoir établi un raccourci dans la description de l'étage dont, en effet, le salon était à l'italienne. On ne peut pas, sur cette erreur, invalider l'ensemble du texte qui, par ailleurs, ne semble pas être mis en défaut.

Ainsi, les mesures et le détail de l'architecture sont conformes aux dessins gravés par Jean Marot qui ont été réalisés vers $1650^{24}$. Les gravures sont nettement postérieures à la construction de la grotte mais elles datent de l'époque où les héritiers d'Albert de Gondi possédaient encore la propriété (Fig. 1). Ce sont les plus anciens documents graphiques connus, toutefois nous ignorons si Marot a fait lui-même (ou utilisé) des relevés contemporains, ou s'il a pu graver à partir de dessins plus anciens. Le plan de la grotte est proche d'un relevé miniature figurant sur un plan des jardins en $1694^{25}$. Toutefois, deux erreurs pourraient faire douter de sa présence sur les lieux : le perron ne correspond pas aux autres sources, plus récentes, avec un escalier à double révolution; par ailleurs, l'escalier intérieur ne se trouvait pas là où il a été gravé, peut-être en raison de la non-inversion de la gravure. Toutefois, les plans des jardins réalisés par les Bâtiments du roi au temps de Louis XIV ne représentent jamais l'escalier menant à l'étage

23. Versailles, Bibl. mun., G 280, p. 111-116). Les mesures ont été soulignées, les termes mentionnant les matériaux ont été mis en gras, les termes décrivant l'architecture et les décors ont été mis en italique.

24. Plan, élévation et profil de la grotte de Noisy, gravures de Jean Marot, publiées vers 1656-1659; Deutsch, 2015, cat. $\mathrm{n}^{\circ}$ 53-55. Une série est conservée par la Bibliothèque nationale de France ; Couzy, 1977, p. 30 (repr.).

25. Montigny-le-Bretonneux, Archives départementales des Yvelines, A 119 ; Couzy, 1977, p. 29 (repr.). 
(auquel on pouvait accéder depuis le parterre haut ${ }^{26}$. Ces indices semblent insuffisants pour rejeter l'exactitude des dessins gravés par Marot, tant la précision des détails du décor est validée par la description de Boulin et par les fouilles.

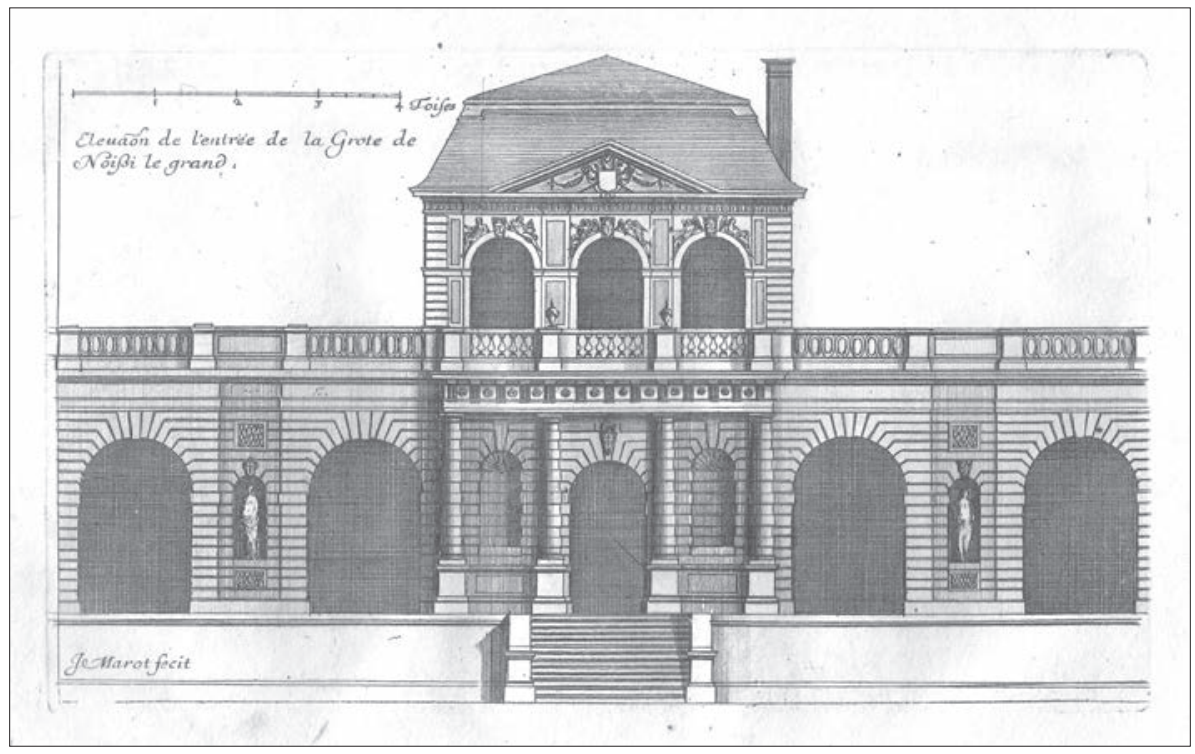

Fig. 1. - Vue du pavillon de la grotte de Noisy, gravure de Jean Marot, vers 1650

Coll. part.

\section{Cliché Bruno Bentz}

Les sources d'archives sont donc plutôt nombreuses et variées, elles apportent des précisions concernant l'implantation de la grotte dans les jardins, l'attribution à son commanditaire, la datation de sa construction et la configuration du bâtiment. Le décor de rocaille et de coquilles, ainsi que les sculptures, la vasque et le jet d'eau sont précisément signalés. Ces données sont vraiment exceptionnelles, notamment pour une grotte restée propriété privée durant près d'un siècle, car l'intégration dans le domaine royal n'a finalement apporté que peu de documents supplémentaires. C'est également une chance de bénéficier d'autant de documents textuels et

26. Arch. nat., $\mathrm{O}^{1} 1904^{1} \mathrm{n}^{\circ} 2$ (1693). Arch. dép. des Yvelines, A 118 (1693) et A 119 (1694); Couzy, 1977, p. 29 et 31 (repr.). 
graphiques quand il est possible de confronter les sources avec les ruines abandonnées lors de la démolition.

\section{Les vestiges}

L'investigation est une aubaine lorsque la démolition d'un bâtiment n'est pas suivie par un nouvel aménagement, sinon un boisement forestier. D'autant que la raison de cette démolition est bien documentée : Louis XV voulait faire araser les bâtiments du château de Noisy pour intégrer le site dans son parc de chasse ${ }^{27}$. Un entrepreneur se chargea donc de récupérer les éléments de maçonnerie qu'il pouvait réemployer, abandonnant sur place ce qui n'avait pas de valeur pour lui et ce qui était inutilement coûteux à détruire. C'est la raison pour laquelle le site a conservé de nombreux vestiges de la grotte, notamment les décors en plâtre et les parties basses du pavillon. Deux campagnes de fouilles ont déjà pu être réalisées : en 2017, un sondage le long de la grotte a permis d'établir un premier diagnostic de son état de conservation (Fig. XII, cahier couleur) ${ }^{28}$; en 2019, une fouille partielle à l'emplacement de l'abside nord a permis de découvrir les vestiges d'une salle complète (Fig. XIII, cahier couleur). Ces résultats permettent également d'établir les conditions d'une intervention sur l'ensemble du bâtiment.

La localisation de la grotte ne pose pas de difficulté, grâce aux plans des jardins réalisés par les Bâtiments du roi à la fin du XVII ${ }^{\mathrm{e}}$ siècle notamment pour des projets de réaménagement des jardins sous la conduite du Premier architecte, Jules Hardouin-Mansart ${ }^{29}$. Ces plans permettent de repérer en négatif in situ le lieu où se trouvait le pavillon car il subsiste aujourd'hui une échancrure dans l'ancien terrassement du jardin haut de la grotte. Par ailleurs, l'ancien promenoir longeant l'ancien mur d'arcades (dont il ne reste que de très rares fondations visibles) existe encore, comme, d'une manière générale, tous les anciens modelés du terrain aménagés à l'époque de Gondi. Ainsi, la grotte a laissé place à un large creux entre le parterre haut et l'ancien promenoir avec un dénivelé en pente douce d'environ

27. Couzy, 1977, p. 32.

28. Bentz, 2018b.

29. Arch. nat., $\mathrm{O}^{1} 1904^{1}$ n$^{\circ} 1$ à 4 (1693). Arch. dép. des Yvelines, A 118 (1693) et A 119 (1694);

Couzy, 1977, p. 29 et 31 (repr.). 
six mètres de hauteur. C'est sous cette butte érodée que se trouvent encore les vestiges de la grotte.

La première fouille a montré que sous une faible épaisseur d'humus (env. 0,5 mètre) se trouvait un épais remblai issu de la démolition de la grotte (de un à six mètres) avec la conservation des structures dans les parties basses, ainsi que bien entendu en sous-œuvre. La fouille de l'abside nord a permis également d'observer l'implantation du bâti, dans le terrassement duquel a été découverte une conduite en terre cuite. Elle servait probablement d'exutoire pour un bassin du parterre haut sans être directement reliée à la grotte. Une fouille complète permettrait de savoir, en outre, si des éléments de la démolition ont été abandonnés à l'extérieur de l'ancien pavillon, notamment en contrebas, du côté du parterre inférieur (lui-même situé environ trois mètres en dessous du niveau de la grotte). La fouille n'a pas encore permis de déceler des remblais provenant de l'étage du pavillon, parmi lesquels doivent notamment se trouver des fragments des peintures à fresque. La période d'occupation de la grotte étant limitée dans le temps, l'absence de travaux attestés au-delà de la période de construction, la démolition ayant été organisée dans un délai assez court, il s'ensuit que la couche de remblais peut être datée de 1732 avec des éléments datant de l'époque de la construction vers 1582 .

Les parties disparues (en dehors de l'étage) sont en partie restituables, grâce notamment aux vestiges des parois et de la voûte retrouvés dans les remblais de démolition (Fig. XIV, cahier couleur). La fouille de l'abside nord avait été décidée en raison de son emplacement à flanc de terrasse, car la hauteur des remblais - et donc des maçonneries encore en place - pouvait être la plus importante. En effet, les maçonneries de l'assise sont encore en place sur plusieurs mètres de hauteur mais c'est un bloc massif, détaché de la structure et à peine effondré qui a conservé des éléments bâtis des parties hautes (environ quatre mètres) d'une absidiole formant un cul-defour. Par ailleurs, provenant également de l'une des trois absidioles de cette salle, c'est le décor apparent d'un cul-de-four qui a été retrouvé, en partie effondré mais en un seul morceau principal, avec son décor bien conservé. Quant à la voûte, de nombreux morceaux du décor en plâtre formé de caissons ont été récupérés. Ainsi, les maçonneries en place permettent de dresser une partie du plan et de l'élévation de la grotte : ces relevés valident en grande partie le dessin gravé par Marot qui peut, dès lors, servir de modèle pour la restitution du bâti et des décors. 
Les caissons de voûte à motif de pavages carrés tronqués (combinant des octogones et des carrés) sont représentés sur la coupe de la grotte en deux endroits : dans le passage de l'entrée vers le salon central puis dans le passage du salon vers l'abside nord. Deux sortes de caissons ont été découvertes avec pour seule différence le traitement décoratif. Ainsi, tous les caissons sont ornés de rosaces, celles à double rangée de cinq pétales au milieu des octogones, celles à sept pétales au milieu des carrés; les plates-bandes d'encadrement sont moulurées avec des cannelures. Parfois, le plâtre est peint en rouge sur toute la surface avec des éléments de dorure sur certaines parties courbes des plates-bandes et sur les pétales des rosaces. La répartition stratigraphique de ces caissons semble indiquer que seule la voûte du second passage (vers l'abside) était peinte et dorée. Une analyse au microscope optique a permis d'observer l'application de trois feuilles d'or successives sur un liant protéique $^{30}$. La présence de cette dorure est exceptionnelle dans un décor de grotte, mais elle apportait des éclats de brillance certainement remarquables.

Le décor du cul-de-four de l'absidiole dessine une coquille Saint-Jacques à l'aide d'innombrables petites coquilles d'un petit bivalve de couleur jaune, la donace, posées alternativement sur leur face interne dans les creux et sur leur face externe dans les côtes. Le support en plâtre, appliqué sur la maçonnerie de pierre, est armé avec de gros silex dans la partie en surplomb. La base du bloc est soulignée par une tablette horizontale, au-delà de laquelle le décor de coquilles se prolonge par des petites rocailles (meulières). Cette partie inférieure était fixée à la maçonnerie par une armature de briques et de tuiles plates liées au plâtre. Le bloc qui a été récupéré avait fait une chute d'environ deux mètres, la zone de contact s'est brisée et un morceau mesurant $1,7 \times 1,5$ mètres s'est posé sans trop de dommages. La fouille du remblai sur lequel il a été retrouvé a montré que la chute de ce bloc était postérieure à la démolition de la voûte voisine, avec une couche relativement stérile sur environ un mètre d'épaisseur : cette partie a donc été détruite après un début de comblement des ruines, sans doute pour achever d'aplanir le terrain. Son extraction a nécessité une consolidation structurelle de la face externe et la mise en place d'un échafaudage en raison de sa masse (environ 500 kilogrammes) ${ }^{31}$.

\section{Pingaud, 2018.}

31. Travaux réalisés sous la conduite d'Hugues de Bazelaire, sculpteur-restaurateur (Versailles). Le bloc a été transporté au Musée national de la Renaissance (Écouen), dépositaire final des vestiges de la grotte de Noisy. 
La mise au jour des décors encore en place dans la partie inférieure de la grotte est le résultat le plus spectaculaire de ces fouilles. En effet, malgré la démolition, certaines maçonneries ont été laissées en place sous le niveau final d'arasement, conservant parfois l'ancien décor. Lors du sondage réalisé en 2017, c'est principalement une pièce de service qui avait été dégagée, mettant au jour des murs de plâtre sans décor. Toutefois, en limite de fouilles, une courte section de la périphérie du salon central, formant une absidiole à trois niches, avait également été découverte. Cet espace avait conservé son décor sur un linéaire d'environ deux mètres, avec des motifs d'architecture : des corbeilles avec cadre de stuc rempli de rocailles (calcite, meulière) et d'une coquille nacrée (ormeau) sous un amas de silex, des palmes dessinées par des coquilles (moules) rayonnant depuis une coquille Saint-Jacques. Le soin apporté à la réalisation de ces décors est souligné par la fixation des blocs de plâtre à l'aide de longs clous en fer à tête ornée et par l'utilisation de plâtre-colle teinté pour unifier les coloris des matériaux décoratifs : blanc pour les plâtres, noir pour les moules ou les pierres noires, rouge pour les meulières rouges, ocre pour les coquilles Saint-Jacques et les rocailles jaunes ${ }^{32}$.

La fouille de 2019 a révélé le décor du pourtour de l'abside nord, conservé (avec des lacunes) sur un à deux mètres de hauteur (l'absidiole ouest n'a pas pu être fouillée, elle répétait probablement le décor des absidioles nord et est) : des niches à dosseret compartimenté encadrées de bandes verticales sont ornées de coquilles et de rocailles, elles s'appuient sur une tablette au-dessous de laquelle se détachent des consoles avec un décor végétal encadrées par des palmes semblables à celles déjà observées dans le salon (moule et coquille Saint-Jacques). Dans les angles entre les absidioles, des compartiments de rocailles (noir, rouge) et de coquilles (bucardes à papilles, ormeaux, moules) forment notamment des motifs floraux. Ces motifs et ces agencements n'étaient pas décrits ou représentés dans les archives ${ }^{33}$.

Les vestiges retrouvés dans les remblais de démolition complètent encore ces données. De très nombreux fragments de plâtres muraux ont été récupérés, parfois simplement moulurés, le plus souvent ornés de coquilles et de rocailles. Certains vestiges ont été découverts en connexion avec le bloc décoré du cul-de-four, leur emplacement peut donc être restitué.

32. Le Dantec, 2019, p. 164-167.

33. Les vestiges en place ont été protégés puis remblayés après les fouilles. 
D'autres vestiges sont analogues aux décors en place et peuvent également être localisés dans la grotte. Il sera plus difficile de restituer la totalité des moulures, corniches, cadres et compartiments, bien que parfois, des éléments de jonction aient été trouvés et que ce décor ait été agencé avec beaucoup de symétrie et de régularité. Une vingtaine de motifs ont déjà pu être identifiés ${ }^{34}$. L'inventaire des matériaux employés est extrêmement varié, tant en minéral avec les roches (grès, galet, meulière, maërl, calcite, tuf, sile $\mathrm{x}^{35}$ ) et les diverses variétés de plâtres, qu'en espèces animales avec une grande variété de mollusques, soit des gastéropodes marins (ormeau, cérithe, lambi, turritelle, murex, pourpre, nasse réticulée, bigorneau), des bivalves marins ou de rivière (coquille Saint-Jacques, pétoncle, spisule, coque, bucarde à papille, moule, deux moules d'eau douce), divers mollusques terrestres ou de rivière ${ }^{36}$.

Plusieurs fragments sculptés ont été mis au jour. En 2017, c'est une pierre d'ornement mural à décor de congélation dont on reconnaît le dessin sur la vue en coupe de Marot du côté de l'entrée, à proximité de l'endroit où elle a été trouvée. En 2019, ce sont des fragments de statuaires en plâtre ou en pierre qui ont été trouvés, en hauts-reliefs ou en bas-reliefs. Ils proviennent de personnages, dont un visage barbu qui pourrait être l'un des mascarons figurés par Marot sur les arches des voûtes, deux têtes d'enfants, des mains et un bras, une épaule, un buste... Par ailleurs, correspondant davantage aux figures animales ou fantastiques décrites par Boulin, de nombreux fragments de formes modelées recouvertes de coquilles de nacre taillée (ormeau) ont aussi été récupérés, provenant soit de la vasque centrale soit des panneaux muraux.

Les fouilles ont aussi permis de dégager plusieurs sols de la grotte. Dans la pièce de service, un carrelage en terre cuite a été observé mais il était recouvert d'une couche de sable jaune égalisant le sol jusqu'au niveau du seuil, légèrement plus haut que le pavement, témoignant donc d'une reprise de construction. Vers le vestibule d'entrée, une dalle de seuil en pierre était encore en place ; dans le salon, quelques petits galets scellés dans un mortier étaient encore visibles et rappelaient la description de Boulin; dans le vestibule vers l'abside, ces incrustations de galets étaient mieux conservées

34. Hori, 2019 (fouilles 2017 ; étude en cours pour les fouilles 2019).

35. Fargeau et Fischer, 2019.

36. Dupont, 2019. 
et formaient des motifs palmés réguliers ; quant au sol de l'abside, un carrelage de petits pavés noirs et blancs était encadré de plaques de pierres plates sur le pourtour et dans le cadre central autour de l'emplacement de la vasque. Ce revêtement est conforme à la description de Boulin en 1732. Cependant, le sol a nécessairement été repris à une date inconnue. En effet, le dégagement des conduites hydrauliques souterraines a révélé que le sol avait été refait au moins à l'emplacement où les tuyaux avaient été arrachés, le remblai de comblement du sol comprenant notamment des fragments de décor (Fig. XV, cahier couleur).

En effet, deux tuyaux en plomb ont été découverts encore en place, entre le vestibule et l'abside : ils avaient été coupés, par arrachement, sous le seuil. Il ne restait ensuite, jusqu'au centre de l'abside, que l'empreinte des tuyaux dans leur ancien support maçonné au-delà duquel ils étaient reliés à la vasque, ce qui explique l'absence de carrelage au centre de la pièce. Il semble peu probable que le sol ait été proprement refait au-dessus de l'arrachage au moment de la démolition de la grotte, il s'agit donc d'une intervention plus ancienne à une époque où la grotte pouvait encore être visitée, mais où le jet d'eau ne fonctionnait plus. D'ailleurs, le démolisseur ne semble pas avoir cherché à récupérer le plomb puisqu'un morceau de tuyau de même aspect et également de 3 centimètres de diamètre extérieur avait été retrouvé en 2017 parmi les remblais de la démolition ${ }^{37}$. Quant à la présence de deux tuyaux en parallèle, elle s'explique par l'arrivée d'une conduite sous pression pour former le jet de la grotte et un départ de conduite, également sous pression, vers le bassin du parterre bas de la grotte pour former un nouveau jet.

Dans le cas de cette grotte du XvI ${ }^{\mathrm{e}}$ siècle, on constate que toutes les sources doivent être mises à contribution pour rétablir l'histoire du bâtiment et des décors, et restituer sa configuration. L'enquête archivistique contribue de manière décisive à attribuer et à dater le pavillon, mais aussi à localiser le site ; elle permet aussi de confronter la description et les représentations à la réalité des vestiges que l'investigation archéologique relève parmi les ruines d'une démolition. Il demeure néanmoins quelques inconnues et des incertitudes, lorsque les données sont lacunaires ou difficiles à interpréter, laissant encore une place aux découvertes par la poursuite des fouilles. L'état dans lequel se trouve la grotte de Noisy présente néanmoins

37. Bentz 2018a, p. 89 et 91 (repr.). 
quelques avantages sur d'autres grottes de cette époque. À la suite de sa démolition, l'ensemble des décors a été scellé sous ses remblais, limitant donc sa période d'utilisation et éliminant tout risque de réparation ou de restauration peu ou mal documentées après cette date. Dans sa dernière époque, au temps où elle appartenait à la couronne, les archives sont nombreuses, notamment les paiements dans les comptes des Bâtiments du roi - aucun document ne mentionne une quelconque intervention. Pour la période précédente, la grotte appartenait à un particulier et les archives sont nécessairement plus rares et incomplètes. Toutefois, un long mémoire de travaux, effectués entre 1659 et 1661 dans la propriété, a été conservé : il mentionne des travaux de pavage aux abords de la grotte mais aucune intervention à l'intérieur. Ainsi, hormis les observations faites ponctuellement sur le sol et les conduites, il semble que l'état dans lequel la grotte a été démolie corresponde à celui de sa construction vers 1582 à l'époque de Gondi. Un autre avantage inhérent à son état de démolition, est la possibilité d'observer les substructions du pavillon, la structure et la composition des décors, enfin la possibilité de disposer d'une quantité de matériaux à analyser relativement infinie. Ainsi, même si on peut toujours déplorer la perte d'un décor probablement parmi les plus originaux de son temps, il est toujours plus intéressant et scientifiquement plus enrichissant de disposer d'un ouvrage archéologiquement (presque) complet.

\section{Sources}

Florence, Archivio di Stato di Firenze [Archives d'État], Mediceo del Principato, 1581-1583, n 4611, fol. 113 bis.

Montigny-le-Bretonneux, AD Yvelines

- A 118, plan du parc de Noiy, sans date [1693].

- A 119, plan des jardins de Noisy, sans date [1694].

Paris, Archives nationales

- $\mathrm{O}^{1} 1904^{1} \mathrm{n}^{\circ} 1$ à 4, plans du parc de Noisy, 1693-1694.

- $\mathrm{O}^{1} 3932^{\mathrm{A}}$, « Du 20 may 1676. Adjudication faite au profit du Roy des terres et seigneuries de Marly le Châtel, Noisy et Bailly, et autres héritages, saisis réellement sur messire François Bossuet, à la requête de messire Jean de Villemont, moïennant la somme de cent-soixante-un-mil livres ", [titre + 194 p.], copie datée du 5 août 1678 de l'acte original rédigé par les notaires Ferret et Beauvais à Paris. 
Paris, BnF, département des Estampes et de la photographie, 4-HA-7 (A), Plan, élévation et profil de la grotte de Noisy, gravures de Jean Marot, pl. 45-47, https://gallica.bnf.fr/ark :/12148/btv1b84965642.

Potsdam, Stiftung Preußische Schlösser und Gärten Berlin- Brandenburg, Graphische Sammlung (ehem. Technische Hochschule Berlin-Charlottenburg, Kriegsverlust) [Potsdam, Fondation des châteaux et jardins prussiens, Collection graphique (anciennement École de technologie de Berlin-Charlottenburg, perte de guerre)], Christoph Pitzler, Reisetagebuch [Carnet de voyage], 1686.

Vatican, Bibliothèque apostolique, Reg.lat.666, fol. 58r, https://digi.vatlib.it/ view/MSS_Reg.lat.666/0131.

Versailles, Bibliothèque municipale, G 280, p. 93-117, [François Bernard Boulin], "Description du chasteau de Noisy dans le grand parc de Versailles, entièrement démoly sur la fin de l'année 1732 ».

Deutsch Kristina, Jean Marot. Un graveur d'architecture à l'époque de Louis XIV, De Gruyter, Berlin, 2015, p. 117-124, cat. p. 437-449, n 53-55.

Forsil Madeleine (dir.), Journal de Jean Héroard, Centre de recherche sur la civilisation de l'Europe moderne, 2 volumes, Paris, 1989.

[Dezallier d'Argenville Antoine-Joseph, Leblond Jean-Baptiste Alexandre], La théorie et la pratique du jardinage, Jean Mariette, Paris, 1709.

Matthieu Pierre, Histoire de France et des choses mémorables advenues aux provinces éstrangères durant sept années de paix, du règne du roy Henri IIII, roy de France et de Navarre, divisée en sept livres, livre 6", Mettayer J. et Guillemot M., Paris, 1605.

\section{Bibliographie}

Bentz Bruno, "L'eau dans les châteaux de plaisance : Marly et Noisy ", Archéologie francilienne, actes des Journées archéologiques d'Île-de-France, Paris, 14-15 décembre 2017, Direction régionale des affaires culturelles d'Îlede-France (Service régional de l'archéologie), Paris, 2018a, p. 77-92.

Bentz Bruno, "Le décor de la grotte de Noisy : résultat des fouilles de 2017 ", Opus Incertum, vol. 4, 2018b, p. 126-135, http://www.fupress.net/index.php/ oi/article/view/25313.

Bentz Bruno, "Les jardins du château de Noisy ", dans Gaugain Lucie, LiÉvaux Pascal et Salamagne Alain (dir.), La fabrique du jardin à la Renaissance, Presses universitaires François-Rabelais, Tours, 2019, p. 115-128.

Bentz Bruno, "La grotte de l'hôtel de Retz à Paris (1578) " et " La grotte de Noisy (1582) ", dans Calafati Marco, Ville e giardini dei Gondi in Francia $i$ castelli di Versailles e Noisy nel Cinquecento, Leo S. Olschki, Florence, 2020 (à paraître). 
Calafati Marco, "Tra Firenze, Lione e Parigi. Architettura e committenza dei Gondi nel beau XVI $\mathrm{e}^{\mathrm{e}}$ siècle ", thèse de post-doctorat, École pratique des hautes études, Paris, sous la direction de Sabine Frommel, 2015.

Cicali Giulia, "Le sculpteur Francesco Bordoni, collaborateur des Francini ", Bulletin monumental, t. 175-4, 2017, p. 357-367.

Couzy Hélène, «Le château de Noisy-le-Roi », Revue de l’art, n³ 38, 1977, p. 23-34.

Dupont Catherine, "Bilan préliminaire des mollusques marins découverts dans la grotte des jardins du château de Noisy : de leur acquisition à leur utilisation (Noisy-le- Roi, Bailly, Yvelines) », CNRS (UMR 6566, Centre de Recherche en Archéologie, Archéosciences, Histoire), Université Rennes 1, septembre 2019.

Fargeau Marc, Fischer Anne-Clothilde, "Géoarchéologie: Analyse pétrographique des éléments décoratifs de la grotte du château de Noisy et interprétation des sources ", mémoire d'initiation à la recherche, sous la direction de Cyril Gagnaison et Jean-David Vernhes, Institut polytechnique UniLaSalle, Beauvais, mars 2019.

Hori Akane, «L'étude des décors architecturaux de la grotte artificielle de Noisy : techniques de construction, typologie, restitutions (XVI $-\mathrm{XVII}^{\mathrm{e}}$ siècle) ", mémoire de Master 1, sous la direction de Florence Journot, Université Paris 1 PanthéonSorbonne, juin 2019.

Jullien de Pommerol Marie-Henriette, Albert de Gondi maréchal de Retz, coll. "Travaux d'humanisme et Renaissance ", n 5, Librairie E. Droz, Genève, 1953.

Le Dantec Tiffanie, «Les façades enduites au plâtre d'Île-de-France. Le déclin du plâtre extérieur, du XVII ${ }^{\mathrm{e}}$ au $\mathrm{Xx}^{\mathrm{e}}$ siècle ", thèse de doctorat, sous la direction de Nadia Hoyet et Jean-Claude Yon, Université Paris-Saclay, 2019.

Le Dantec Tiffanie, Bentz Bruno, Bolle Caroline et al., "Plaster nails: an unexplored aspect of construction history ", dans CAmpbeLl James W. P. et al. (éd.), Water, Doors and Buildings, Construction History Society, Cambridge, 2019, p. 313-329.

MaQuet Adrien, "Notice sur le château et sur le couvent des Cordeliers de Noisy-le-Roi ", Mémoires de la Société des sciences morales des lettres et des arts de Seine-et-Oise, t. XI, 1878, p. 349-380.

Odier Jeanne, "Voyage en France d'un jeune gentilhomme morave en 1599 et 1600 », Mélanges d'archéologie et d'histoire, t. 43, 1926, p. 140-173.

Pingaud Nathalie, rapport n ${ }^{\circ} 39421$ (analyse de la dorure), Centre de recherche et de restauration des musées de France, décembre 2018.

TAмвour Ernest, Les Gondi et le château de Noisy-le-Roi, 1568-1732 (complété et publié par Liliane Pierre-Bonnet), Paris, 1925.

Terrade Albert, "Le château et le village de Noisy-le-Roi ", Versailles illustré, t. III, avril 1898, p. 29-33 et mars 1899 , p. 80-84 ; " Le château de Noisyle-Roi (documents nouveaux) ", Versailles illustré, t. IV, avril 1899, p. 6-9. 


\section{L'auteur}

Bruno Bentz est docteur en archéologie (université de Paris-Sorbonne). Ses travaux portent sur la période moderne et contemporaine. Il a dirigé des fouilles archéologiques à Marly, Versailles puis désormais à Noisy. Ses publications ont été consacrées aux carrelages en faïence et à la verrerie, au mobilier et à la peinture, aux bains et aux globes, à la télégraphie et à l'hydraulique, aux jardins et aux fosses d'aisances... où l'archéologie privilégie l'analyse technique et les processus ergologiques de la fabrication aux moyens et techniques d'observation. Il a fondé et préside depuis 1989 l'association OMAGE. Contact : brunobentz@ free.fr 